\title{
Qualidade de Vida nas Epilepsias Refratárias
}

\author{
Neide Barreira Alonso* \\ Marly de Albuquerque**
}

\begin{abstract}
RESUMO
O objetivo deste trabalho é ressaltar a importância dos instrumentos que permitam avaliar a qualidade de vida de indivíduos com epilepsia. Para tanto, apresentamos um resumo breve de oito inventários utilizados em epilepsia. Acentuamos a necessidade de estudos que objetivem a tradução e avaliação das propriedades psicométricas dos questionários já validados internacionalmente, o que possibilitará a comparação com os dados de nosso país. A mensuração do impacto das crises epilépticas é fundamental para a programação terapêutica e inserção social de indivíduos com epilepsia.
\end{abstract}

Unitermos: Qualidade de vida, epilepsias refratárias, tradução e validação de questionários.

\section{Introdução}

Os avanços tecnológicos e a velocidade dos meios de comunicação transformaram a relação do homem com o tempo.

As distâncias tornaram-se praticamente inexistentes, as informações chegam a todo instante aos milhares, a interação do homem com seu trabalho, família, relações interpessoais e sociais também sofreu considerável impacto.

Em meio a esse turbilhão, atravessado pela quantidade de estímulos o homem parece voltar-se para a qualidade da experiência vivida, aspecto diferencial em cada esfera da vida, que possibilita imprimir sua marca no tempo. Nasce a preocupação com a qualidade de vida.

A origem do conceito de qualidade de vida surgiu após a $2^{\text {a }}$ Guerra Mundial, quando a prosperidade econômica e o aumento do padrão de vida associam a satisfação, o bem-estar e a realização psicológica com vários aspectos da vida ${ }^{1}$.

A apreciação subjetiva da qualidade de vida tem sido considerada como o julgamento - com maior ou menor satisfação - que um indivíduo faz sobre diferentes áreas de sua vida ${ }^{1}$.

Nas três últimas décadas proliferaram as definições sobre qualidade de vida, porém não há um consenso sobre a definição mais adequada. Podemos conceituar qualidade de vida como sendo a percepção de um indivíduo de sua posição na vida, no contexto do sistema de valores e da cultura em que vive e em relação às suas metas, expectativas, padrões e interesses. Trata-se de um conceito amplo que agrega saúde física, aspectos psicológicos, nível de independência, sociabilidade e relações com o ambiente.

\section{Impacto das epilepsias na qualidade de vida}

Grande parte dos estudos sobre qualidade de vida está voltada para a compreensão da vida de pessoas acometidas por alguma doença, principalmente as crônicas que muitas vezes obrigam os indivíduos a conviver com sintomas limitantes.

No caso das síndromes epilépticas, as repercussões das crises se fazem sentir no cotidiano do paciente, pois interferem direta e negativamente na qualidade de

\footnotetext{
* Psicóloga da Unidade de Pesquisa e Tratamento das Epilepsias da Universidade Federal de São Paulo, Escola Paulista de Medicina, UNIFESP.

** Neurologista, Professor Adjunto de Neurologia da Universidade de Mogi das Cruzes, Presidente da Associação Brasileira de Epilepsia.
} 
vida dessas pessoas. Essa interferência pode ser decorrente tanto da frequiência e gravidade das crises, quanto da duração da síndrome epiléptica ${ }^{2-4}$.

Com o tratamento médico adequado, cerca de $75 \%$ dos pacientes têm suas crises controladas; os demais podem se beneficiar dos tratamentos cirúrgicos. A cirurgia é um tratamento efetivo e mais barato que os tratamentos medicamentosos a longo prazo, devendo portanto ser considerada precocemente, mesmo em países em desenvolvimento ${ }^{5}$. Os custos totais, que incluem também a avaliação pré-operatória, variam de $1.200^{6}$ a 5.020 dólares $^{7}$; além do que leva a um processo de ajustamento, onde os pacientes reaprendem a viver bem $^{8}$.

Os pacientes com epilepsia do lobo temporal, refratárias aos tratamentos medicamentosos, são candidatos potenciais para a lobectomia temporal anterior (LTA) e a abordagem cirúrgica, quando bem indicada, pode eliminar ou reduzir a frequiência de crises epilépticas em $70 \%$ dos casos.

Devem ser usados protocolos para a avaliação do custo/benefício do tratamento medicamentoso e procedimento cirúrgico desses casos. Pode ser utilizado o modelo de Markov, que avalia: a freqüência de crises no pós-operatório, a qualidade de vida, morte decorrente da cirurgia ou causas naturais, custos médicos diretos, associados com avaliação e tratamento ambulatorial desses pacientes, hospitalização e cirurgia ${ }^{9}$.

O custo da LTA é comparável a outros procedimentos cirúrgicos, como artroplastia total de joelho ou angioplastia. Os resultados são dependentes da ocorrência ou não de crises e da melhor qualidade de vida. Ainda são necessários estudos para avaliar a qualidade de vida após a lobectomia, pois o impacto da cirurgia na qualidade de vida não tem sido bem documentado ${ }^{10-13}$. A diminuição ou remissão das crises que se segue à cirurgia nem sempre é acompanhada de um retorno ao trabalho ou de uma ampliação do círculo social e dos vínculos interpessoais do paciente ${ }^{12}$, embora Rao e Radhakrishnam ${ }^{6}$ tenham relatado que 119 pacientes submetidos a LTA obtiveram melhores empregos e melhor qualidade de vida, concluindo que a LTA é a melhor opção custo/benefício que os tratamentos convencionais.

Devem ser criados programas para cirurgia de epilepsia em países em desenvolvimento, visto que o custo/benefício da LTA é bastante aceitável, mesmo quando muitos parâmetros são levados em consideração. São necessários estudos prospectivos multicêntricos para melhor avaliação dos períodos sem crises e seus efeitos na qualidade de vida. Também são necessários estudos dos resultados dos tratamentos medicamentosos no controle das crises e seu impacto na qualidade de vida ${ }^{14}$.

No início da década de 1980 começaram a surgir trabalhos voltados para a criação de instrumentos de avaliação de qualidade de vida, considerando as particularidades das síndromes epilépticas. Foram desenvolvidos estudos quantitativos da população que trouxeram informações relevantes sobre a qualidade de vida de pessoas com epilepsia.

Em 1992, a Liga Internacional de Epilepsia promoveu um evento em Portugal, com a finalidade de discutir a questão da qualidade de vida em pessoas com epilepsia e criar instrumentos que permitissem sua mensuração. Esses inventários deveriam ser padronizados e as suas propriedades psicométricas testadas ${ }^{15}$.

\section{Aspectos envolvidos na tradução e validação dos instrumentos}

A grande maioria dos instrumentos de avaliação de qualidade de vida foi formulada na língua inglesa e, por isso, direcionada para ser utilizada em população que fala esse idioma. Devido ao crescente número de ensaios multicêntricos, criou-se a necessidade de desenvolver-se medidas delineadas especialmente para utilização em países cujo idioma não seja o inglês.

Isso pode ser feito de duas maneiras: desenvolvendo-se uma nova medida ou modificando e adaptando uma medida previamente validada em outra língua, baseando-se num processo de adaptação cultural.

A primeira opção demanda uma quantidade maior de tempo e empenho tanto pessoal como financeiro para proceder-se à criação de um novo instrumento, adequando novos conceitos, selecionando seus itens e testando sua validade ${ }^{16}$.

Desenhar um novo instrumento ainda implica em que este possa ser reproduzido em populações diferentes com a mesma patologia e confirmadas as suas propriedades psicométricas.

Tentar uniformizar uma linguagem criada para mensurar o impacto de uma doença na qualidade de vida é um processo muito mais enriquecedor, porque pressupõe estudos mais consistentes de um dado instrumento, do que constatarmos que inúmeras pessoas se utilizam de escalas diversas para representar o mesmo universo em questão.

Além disso, é importante avaliar a real necessidade de criar-se um novo instrumento, caso exista um anterior com a mesma proposta e de boa qualidade ${ }^{16}$.

$\mathrm{Na}$ segunda opção, entretanto, não basta que o instrumento seja simplesmente traduzido. 
É necessário que se faça uma avaliação rigorosa de sua tradução e adaptação transcultural, bem como a confirmação de suas propriedades de medida após a tradução ${ }^{17}$.

Os passos envolvidos nesse processo compõemse de:

- Tradução.

- Adaptação cultural.

- Adaptação das propriedades psicométricas: confiabilidade e validade.

Para melhor visualização desse processo, veja anexo 1.

\section{Tradução}

A back-translation ou retrotradução é uma das técnicas mais utilizadas de tradução. Esse método implica em cuidados especiais na fase de tradução, visando preservar o significado da versão original do instrumento em cada detalhe da tradução para o novo idioma. Requer nessa fase dois tradutores bilíngües, ou seja, que tenham familiaridade com os dois idiomas envolvidos na tradução, havendo necessariamente convivido nas duas culturas ${ }^{1}$.

Esse tipo de tradução evita a utilização do significado literal de um termo, considerando os aspectos culturais que possam estar a ele relacionados e efetuando as adaptações necessárias para que o sentido original seja preservado ${ }^{17}$.

O instrumento, uma vez traduzido deve ser vertido para o idioma original e o resultado comparado ao instrumento de origem. Essa etapa deve ser realizada por dois outros tradutores que apresentem um bom conhecimento dos dois idiomas, embora nessa fase a tradução deva ser feita para a língua materna dos tradutores nativos ${ }^{16}$.

\section{Adaptação cultural}

Envolve o processo de tradução, considerando-se as particularidades da cultura para a qual o instrumento foi traduzido. Alguns aspectos devem ser verificados:

Equivalência semântica: baseia-se na equivalência gramatical e de vocabulário, procurando-se manter o significado de cada item do instrumento após a tradução para outra língua ${ }^{1,16}$.

Equivalência conceitual: o instrumento irá medir o mesmo construto básico, tanto na cultura de origem do instrumento, quanto na cultura para o qual ele foi traduzido ${ }^{1}$.

Equivalência de conteúdo: cada item do instrumento deve representar adequadamente o fenômeno investigado em diferentes culturas ${ }^{17}$.
Equivalência de critério: objetiva que as normas de interpretação dos dados obtidos sejam as mesmas, independentemente da cultura ${ }^{17}$.

Equivalência técnica: enfatiza a necessidade de se manter o mesmo método de coleta de dados em qualquer cultura ${ }^{17}$.

Equivalência experimental ou cultural: os termos utilizados no instrumento devem ser coerentes com as experiências vividas pela população a que se destina, dentro de seu contexto cultural ${ }^{16}$.

\section{Avaliação das propriedades psicométricas}

A extensão na qual um indicador empírico representa o conceito subjacente é avaliada através de duas propriedades psicométricas básicas: a validade e a confiabilidade $^{18}$.

Confiabilidade: confiabilidade significa que medidas avaliadas dos mesmos indivíduos em diferentes ocasiões, ou por diferentes observadores, ou por testes paralelos ou similares devem produzir resultados iguais ou similares ${ }^{19}$.

Os métodos mais comumente utilizados para se estimar a confiabilidade são: teste-reteste e consistência interna.

Confiabilidade teste-reteste: é estimada através da correlação entre a administração do teste e a mesma amostra em duas ocasiões diferentes. Quanto menor o período entre as aplicações, maior a correlação, variando de 2 a 14 dias. Os índices de confiabilidade teste-reteste aceitáveis são os maiores que $0,70^{1}$.

Consistência interna: refere-se à consistência das respostas do indivíduo aos itens do instrumento. É realizado numa única aplicação e a confiabilidade é estimada através de coeficientes de correlação ${ }^{1}$. Desde a década de 1950, o coeficiente mais utilizado para estimar a consistência interna de uma escala é o alpha de Cronbach.

O coeficiente alpha de Cronbach é uma medida que avalia a consistência interna dos itens do instrumento, ou seja, o quanto os itens de um instrumento estão relacionados entre $\mathrm{si}^{17}$. Esse índice, como todos os coeficientes de confiabilidade podem variar de 0,00 a 1,00. Escalas com índices inferiores a 0,70, devem ser evitadas.

\section{Validade}

A validade de um instrumento se refere à capacidade da medida em medir o fenômeno a que ela se propõe a medir ${ }^{1}$. Os métodos pelos quais torna-se mais viável confirmar a validade de um instrumento são a validade de construto, a validade de conteúdo e de critério. 
Validade de construto: é a análise do significado do escore do teste em termos do conceito. Segundo Cronbach e Meehl ${ }^{20}$ a validade de construto precisa ser investigada sempre que não exista um critério ou que o universo do conteúdo não se mostre adequado para definir a qualidade a ser medida. Trata-se de um processo complexo que avalia efetivamente o quanto a medida mede um conceito teórico específico ${ }^{1}$.

Validade de conteúdo: pressupõe uma descrição detalhada do conteúdo do domínio, avaliando sua representabilidade em relação ao fenômeno em foco.

Validade de critério: se refere à extensão da correspondência entre o instrumento e o critério utilizado $^{18}$. Para a estimativa da validade de critério usa-se o gold-standard ou um critério externo ao qual o critério atual possa ser comparado.

A mais importante limitação desse tipo de validade é que na maioria das ciências sociais e na área da psicologia não existe o padrão-ouro ${ }^{1}$.

A validade de critério em ciências humanas é então aferida segundo um critério externo, com freqüência com um outro instrumento que será comparado em seus diversos domínios àquele que se deseja validar.

Cumpre salientar que os conceitos expostos acima constituem um universo complexo, fruto da interseção entre a estatística, a psicometria e as ciências humanas, com o objetivo de criar formas de mensurar aspectos subjetivos envolvidos na percepção do indivíduo diante da doença.

No Brasil, até o presente momento, não dispomos de literatura sobre tradução, adaptação e validação de escalas de qualidade de vida nas epilepsias.

Esse trabalho é sempre decorrente do envolvimento de uma equipe multidisciplinar voltada para o estudo da qualidade de vida em pessoas com epilepsia que, através de esforços conjuntos, visa disponibilizar instrumentos para avaliar a percepção do paciente em seu processo de saúde/enfermidade.

A partir de uma minuciosa avaliação da qualidade de vida, torna-se possível a implantação de programas de intervenção como grupos de apoio psicopedagógico, grupos de preparo para lobectomia temporal e acompanhamento pós-cirúrgico, bem como executar modificações na conduta terapêutica.

\section{Instrumentos utilizados para avaliação da qualidade de vida nas epilepsias}

Assim como não existe um conceito único para designar o que se entende por qualidade de vida, também não há um instrumento único para avaliá-la.
Os instrumentos utilizados podem ser divididos em dois grupos: genéricos e específicos. Os instrumentos mais usados na avaliação da qualidade vida dos indivíduos com epilepsia são:

- A escala genérica SF-36 (medical outcomes study 36-item short-form health survey).

- WPSI (Washington pychosocial seizure inventory).

- ESI- 55 (epilepsy surgery inventory).

- QOLIE-89 e as formas reduzidas 31 e 10 (quality of life in epilepsy).

- SEALS (side effect and life satisfaction).

- PESOS (performance sociodemographic aspects, subjective evaluation/estimation).

- LSSS (Liverpool seizure severity scale) e bateria de qualidade de vida Liverpool (LQOL).

\section{Instrumentos genéricos}

Os instrumentos genéricos são desenvolvidos com a finalidade de refletir o impacto de uma doença sobre a vida de pacientes numa população. Avaliam aspectos relativos a função, disfunção, desconforto físico e emocional. Podem ser utilizados em qualquer população, entretanto, não são sensíveis para detectar aspectos particulares ou específicos da qualidade de vida de uma determinada doença ${ }^{16}$.

\section{The Medical Outcomes Study 36 item Short-} Form Health Survey (SF-36)

O SF-36 foi criado para ser um questionário genérico de avaliação de saúde, de fácil administração e compreensão ${ }^{21}$. Por ser um questionário genérico, seus conceitos não são tão específicos para uma determinada idade, doença ou grupo de tratamento, portanto permite comparações entre diferentes patologias ou entre diferentes tratamentos ${ }^{22}$.

O questionário SF-36 foi derivado inicialmente de um questionário de avaliação de saúde formado por 149 itens, desenvolvido e testado em mais de 22 mil pacientes, como parte de um estudo de avaliação de saúde.

Com o intuito de formular um instrumento abrangente, mas não tão extenso, chegou-se à forma de 36 questões (Anexo 2).

O SF-36 é um questionário multidimensional, formado por 36 itens, englobados em 8 escalas ou componentes: capacidade funcional (10 itens), aspectos físicos (4 itens), dor (2 itens), estado geral de saúde (5 itens), vitalidade (4 itens), aspectos sociais (2 itens), aspectos emocionais (3 itens), saúde mental (5 itens) e mais uma questão de avaliação comparativa entre as condições de saúde atual e a um ano atrás ${ }^{21}$. 
O SF-36 tem sido um instrumento utilizado para avaliar a qualidade de vida em indivíduos epilépticos. Escalas mundialmente usadas nas epilepsias possuem como componente o SF-36, ao qual foram acrescidas questões específicas sobre epilepsias.

Essa escala tem fornecido informações relevantes sobre a percepção do paciente epiléptico em relação a sua saúde, o que permite avaliar e modificar condutas terapêuticas ${ }^{23}$.

Escores elevados têm sido encontrados em indivíduos epilépticos com crises controladas, assim como adultos epilépticos revelaram piores índices de qualidade de vida, quando comparados a população de um modo geral. Tais resultados indicam e confirmam a sensibilidade desse instrumento para avaliação da qualidade de vida nesses pacientes ${ }^{24}$.

Em 1999, Jacoby et al. ${ }^{25}$ testaram detalhadamente as propriedades psicométricas do SF-36 em portadores de epilepsia e também encontaram índices menores de qualidade de vida em pacientes com crises freqüentes quando comparados àqueles com crises controladas ou com crises pouco freqüentes (Anexo 2).

\section{Instrumentos específicos}

Os instrumentos específicos são capazes de avaliar de forma individual e específica determinados aspectos da qualidade de vida, proporcionando uma maior capacidade de detecção de melhora ou piora do aspecto em estudo. Sua principal característica é seu potencial de ser sensível às alterações (responsiveness), ou seja, a capacidade que possui de detectar alterações após uma determinada intervenção. Podem ser específicos para uma determinada função (capacidade física, sono, função sexual), para uma determinada população (idosos, jovens) e para uma determinada alteração, por exemplo a dor ${ }^{16}$.

\section{Washington psychosocial seizure inventory (WPSI)}

Questionário pioneiro na avaliação da qualidade de vida nas epilepsias foi desenvolvido por Dodrill et al. ${ }^{26}$. Composto por 132 questões, com duas alternativas para as respostas, baseia-se nas seguintes categorias: retaguarda familiar, ajustamento interpessoal, emocional, social e vocacional, status financeiro, ajustamento às crises, à medicação e ao tratamento.

Trata-se de um instrumento bastante abrangente para o levantamento de dados sobre a problemática dos indivíduos com epilepsia, porém revela-se limitado em razão da quantidade de respostas possíveis.

As escalas de qualidade de vida mais utilizadas atualmente são aquelas que permitem algumas possibilidades de resposta, num circuito crescente ou decrescente de intensidade dos estados de saúde/ enfermidade, fato que contribui para maior discriminação e precisão de tais estados.

\section{Epilepsy surgery inventory (ESI-55)}

O ESI-55 foi desenhado por Vickrey et al. ${ }^{11}$, em 1992, com o objetivo inicial de avaliar mudanças na qualidade de vida de pacientes submetidos à lobectomia temporal.

Para isso, utilizou-se o instrumento genérico SF-36 e acrescentou-se a ele 19 itens baseados em aspectos relevantes da vida das pessoas com epilepsia, compondo o ESI-55 (Anexo 3). As 19 questões complementares contidas no ESI-55 dizem respeito às limitações físicas, emocionais, cognitivas e sociais impostas pelas epilepsias ${ }^{11,15}$.

A confiabilidade da escala foi testada utilizando-se o coeficiente alpha de Cronbach, que mostrou índices entre 0,76 a 0,88 para cada subescala.

A validade de construto foi analisada mediante a comparação dos escores obtidos nas subescalas do ESI55 por pacientes submetidos a lobectomia temporal, livres de crises e aqueles que apresentavam crises parciais complexas e crises parciais complexas com generalização secundária. Escores mais elevados foram encontrados em pacientes com crises controladas após o procedimento cirúrgico ${ }^{11}$.

A validade de critério foi confirmada através da correlação entre os resultados obtidos nas subescalas de aspectos físicos e emocionais do ESI-55 e as subescalas que mediam os mesmos aspectos do Mood Profile Instrument ${ }^{27}$.

Wiebe et al. ${ }^{28}$ investigaram a sensibilidade do ESI55 e constataram que as questões relativas à percepção de problemas de memória e bem-estar emocional eram as mais sensíveis para detectar transformações na qualidade de vida dos indivíduos epilépticos.

O estudo realizado por Vickrey et al. ${ }^{12}$ revelou que pacientes submetidos ao tratamento cirúrgico de lobectomia temporal apresentavam escores mais elevados em 5 das 11 escalas do ESI-55 após a cirurgia. Observou-se, entretanto, que a melhoria na qualidade de vida não se fez acompanhar de uma maior inserção das pessoas com epilepsia no mercado de trabalho.

O ESI-55 é um instrumento bastante utilizado na avaliação da qualidade de vida em pacientes candidatos à lobectomia temporal e nos casos de epilepsias refratárias. Pouco investigado, mereceria estudos mais consistentes, visando sua tradução, adaptação e validação para outros países (Anexo 3). 


\section{The quality of life in epilepsy (QOLIE-89)}

Desenvolvido por Devinsky et al..$^{29}$, consiste do instrumento genérico SF-36, ao qual foram acrescidas 48 questões específicas sobre as interferências das crises epilépticas na vida cotidiana, como: dificuldade de atenção, concentração, memória, linguagem, efeitos adversos da medicação, sociabilidade, trabalho, direção de veículos, preocupação com as crises, aspectos emocionais, além de 6 itens adicionais que versam sobre atitudes em relação à epilepsia ${ }^{30,31}$. É o inventário mais utilizado na avaliação das epilepsias leves e moderadas.

O QOLIE-89 auxiliou a marcar uma nova etapa. A abrangência de seus temas avançou na compreensão de como as crises epilépticas afetam a vida das pessoas: na percepção do estigma, na vergonha de apresentar uma crise em público, na inserção no mercado de trabalho e na sociabilidade ${ }^{2}$.

A validade e a confiabilidade do QOLIE-89 têm sido amplamente demonstradas na população americana $^{30}$, bem como a convergência entre esse instrumento e a avaliação neuropsicológica ou outros testes psicológicos ${ }^{31}$.

As formas abreviadas QOLIE-31 e 10 foram empiricamente selecionadas dos 89 itens, baseando-se em questões relevantes da vida das pessoas afetadas por esse distúrbio ${ }^{31}$.

A versão do QOLIE, com 31 itens, é a mais utilizada mundialmente por ser breve e de fácil avaliação ${ }^{32}$. Foi traduzida para os seguintes países: Dinamarca, Holanda, Alemanha, Canadá, França, Itália, Espanha, Suécia e Reino Unido. Seu uso em outros países, no entanto, requer adaptações lingüísticas, culturais e análise de suas propriedades psicométricas ${ }^{30}$.

A forma do QOLIE que contém 10 itens inclui apenas aqueles correspondentes aos aspectos cognitivos e emocionais. Necessita estudos mais detalhados para confirmação de sua validade e confiabilidade ${ }^{31}$.

A limitação do QOLIE deve-se ao fato de exigir do indivíduo um nível de escolaridade alto (10 a 12 anos de estudos), o que dificulta sobremaneira seu uso em pacientes advindos de países em desenvolvimento, com baixos índices de escolaridade.

\section{Side effect and life satisfation (SEALS)}

Desenhado em 1982 para averiguar os efeitos psicossociais causados pelas drogas antiepilépticas (DAE). $\mathrm{O}$ objetivo era construir um instrumento que permitisse distinguir não apenas os efeitos adversos dos medicamentos, daqueles ocasionados pelas epilepsias, mas que também pudesse discriminar efeitos de uma DAE quando em comparação com outras utilizadas em ensaios clínicos ${ }^{33}$.
Gillham et al. ${ }^{34}$ fizeram algumas modificações no instrumento original, que consta atualmente de 38 itens. É um questionário de rápida compreensão e de fácil aplicação.

Suas propriedades psicométricas têm sido testadas e o SEALS tem se revelado um instrumento sensível para captar diferenças em relação às várias terapêuticas medicamentosas propostas, mostrando-se útil na avaliação do impacto das DAE na qualidade de vida das pessoas com epilepsia que participam de ensaios clínicos multicêntricos ${ }^{33}$.

\section{Performance, sociodemographic aspects, subjective evaluation/ estimation (PESOS)}

O PESOS foi produzido no serviço de epilepsias de Bethel, Alemanha, e aplicado a 509 pacientes com epilepsias de difícil tratamento.

Foi desenhado com o objetivo de avaliar a gravidade das epilepsias, a percepção das pessoas epilépticas sobre sua própria qualidade de vida, identificar restrições na vida cotidiana, problemas psicossociais e organizar formas de intervenção através de grupos de aconselhamento e apoio aos pacientes.

O PESOS permite a avaliação da qualidade de vida em diferentes grupos de indivíduos: aqueles com crises refratárias à terapêutica medicamentosa, pacientes submetidos a tratamentos cirúrgicos, aqueles com dificuldades psicossociais e ocupacionais.

Possui uma dupla função: diagnóstica e avaliativa. Por um lado identifica problemas individuais relacionados às atividades ocupacionais, sociabilidade, mobilidade para execução das atividades de vida diária, adesão ao tratamento, efeitos adversos dos medicamentos; por outro, avalia mudanças significativas ocorridas na vida dessas pessoas após realizar uma intervenção - grupos de aconselhamento e apoio ${ }^{35}$. domínios:

Esse instrumento compreende os seguintes

1. Dados sociodemográficos.

2. Avaliação subjetiva da situação de trabalho.

3. Avaliação subjetiva dos estudos.

4. Avaliação subjetiva da situação na família.

5. Aspectos específicos sobre as crises epilépticas.

6. Avaliação subjetiva da eficácia das medicações e tolerabilidade destas.

7. Mobilidade para realização das atividades da vida diária e lazer.

8. Relacionamentos e suporte social.

9. Restrições na vida cotidiana causadas pela epilepsia. 
10. Adaptação à epilepsia.

11. Estigma.

12. Preocupações específicas sobre epilepsia.

Utilizou-se o QOLIE-31 (versão alemã) como medida de correlação para investigar as propriedades psicométricas do PESOS. Foram analisadas sete subescalas do PESOS: restrições na vida diária atribuídas à epilepsia, preocupações relativas às crises epilépticas, estigma, adaptação emocional à doença, dificuldades no trabalho, estudos e relacionamento familiar.

A correlação entre as subescalas do PESOS e do QOLIE-31 mostrou-se moderada e alta, principalmente nos aspectos que envolveram restrições na vida cotidiana, funcionamento social e preocupações envolvendo as crises epilépticas.

Sua confiabilidade foi analisada através do método teste-reteste em um grupo de 256 pacientes e a consistência interna do PESOS revelou-se satisfatória para todas as subescalas (Alpha de Cronbach > 0,78), exceção feita a subescala de adaptação emocional $(0,65)$.

\section{Liverpool seizure severity scale (LSSS) e bateria de qualidade de vida Liverpool (LQOL)}

A escala de gravidade de crises de Liverpool (LSSS) e a bateria de qualidade de vida Liverpool (LQOL) foram desenvolvidas na Grã-Bretanha ${ }^{36,37}$. A LSSS é um questionário administrado ao paciente com 20 itens e é constituído por duas subescalas: uma escala crítica, com 10 questões para a avaliação dos fenômenos críticos e pós-críticos e uma escala de percepção, relacionada à percepção da aproximação da crise e seu impacto. Os itens incluem eventos objetivos relacionados às crises e eventos subjetivos, relacionados à gravidade de cada crise. Se o paciente apresenta dois ou mais tipos de crises, é então solicitado a aplicar a escala para crises, chamadas major e minor, sendo que a definição entre essas formas é deixada a critério dos pacientes ${ }^{36}$.

Dados de confiança têm sido apresentados no teste-reteste, com valor do coeficiente de correlação de Pearson de 0,8 para as subescalas, e o valor do alfa de Cronbach de 0,85 para a escala crítica e 0,69 para a escala de percepção ${ }^{38}$.

A LQOL consta de seis subescalas: efeitos positivos das drogas, efeitos negativos das drogas, realização pessoal, equilíbrio afetivo, impacto da epilepsia e sense of mastery ${ }^{37}$.

\section{SUMMARY}

\section{Quality of life in refractory epilepsy}

The aim of this article is to emphasize the importance of questionnaires which allow the evaluation of quality of life of people with epilepsy. Eight questionnaires have been used in epilepsy and are briefly described here. Studies emphasizing translation and evaluation of the psychometric properties of instruments already validated abroad are necessary. This will allow the comparison of data among different countries. The measurement of the impact of the seizures is fundamental for therapeutic approach and social insertion of people with epilepsy, particularly of those with refractory seizures.

\section{Keyw ords}

Quality of life, refractory epilepsy, psychometric properties of quality of life instruments.

Anexo 1 Tradução e validação dos instrumentos

\begin{tabular}{ll} 
Tradução: & língua original $\rightleftarrows$ língua desejada \\
& dois tradutores bilíngües \\
& dois tradutores nativos \\
& Semântica: vocabular e gramatical \\
Adaptação cultural: & Conceitual: aferição do construto \\
(equivalência) & Conteúdo: representação do fenômeno \\
& Critério: normas de interpretação \\
& Técnica: método de coleta de dados \\
& Experimental ou cultural: considera as experiências culturais da população \\
& Teste-reteste: correlação na aplicação à mesma amostra em ocasiões diferentes \\
Confiabilidade: & Consistência interna: respostas do indivíduo - coeficiente alpha de CRONBACH \\
Calidade: & construto: aferição do conceito \\
& conteúdo: representabilidade do fenômeno \\
& critério: correspondência com outro instrumento \\
\hline
\end{tabular}


Anexo 2 Escala genérica SF-36

Instruções: Esta pesquisa questiona você sobre sua saúde. Estas informações nos manterão informados de como você se sente e quão bem você é capaz de fazer suas atividades da vida diária. Responda cada questão marcando a resposta como indicado. Caso você esteja inseguro ou em dúvida em como responder, por favor, tente responder o melhor que puder.

1. Em geral, você diria que sua saúde é:

(circule uma)

\begin{tabular}{ccccc}
\hline Excelente & Muito boa & Boa & Ruim & Muito ruim \\
\hline 1 & 2 & 3 & 4 & 5 \\
\hline
\end{tabular}

2. Comparada há um ano atrás, como você classificaria sua saúde em geral, agora?

(circule uma)

\begin{tabular}{ccccc}
\hline Muito melhor & Um pouco melhor & Quase a mesma & Um pouco pior & Muito pior \\
\hline 1 & 2 & 3 & 4 & 5 \\
\hline
\end{tabular}

3. Os seguintes itens são sobre atividades que você poderia fazer atualmente durante um dia comum. Devido a sua saúde, você teria dificuldade para fazer essas atividades? Neste caso, quanto?

(circule um número em cada linha)

\begin{tabular}{lccc}
\hline Atividades & $\begin{array}{c}\text { Sim. Dificulta } \\
\text { muito }\end{array}$ & $\begin{array}{c}\text { Sim. Dificulta } \\
\text { um pouco }\end{array}$ & $\begin{array}{c}\text { Não. Não dificulta } \\
\text { de modo algum }\end{array}$ \\
\hline $\begin{array}{l}\text { Atividades vigorosas, que exigem muito esforço, } \\
\text { tais como correr, levantar objetos pesados, }\end{array}$ & 1 & 2 & 3 \\
participar em esportes árduos & & & 3 \\
Atividades moderadas, tais como mover uma & 1 & 2 & 3 \\
mesa, passar aspirador de pó, jogar bola, varrer & & & 3 \\
a casa & & 2 & 3 \\
Levantar ou carregar mantimentos & 1 & 2 & 3 \\
Subir vários lances de escada & 1 & 2 & 3 \\
Subir um lance de escada & 1 & 2 & 3 \\
Curvar-se, ajoelhar-se ou dobrar-se & 1 & 2 & 3 \\
Andar mais de 1 quilômetro & 1 & 2 & 3 \\
Andar vários quarteirões & 1 & 2 & \\
Andar um quarteirão & 1 & & \\
Tomar banho ou vestir-se & 1 & & \\
\hline
\end{tabular}

4. Durante as últimas 4 semanas, você teve algum dos seguintes problemas com o seu trabalho ou com alguma atividade diária regular, como conseqüência de sua saúde física?

(circule um número em cada linha)

Você diminuiu a quantidade de tempo que dedicava ao seu trabalho ou a outras atividades?

Realizou menos tarefas do que você gostaria?

Esteve limitado no seu tipo de trabalho ou em outras atividades?

Teve dificuldade de fazer seu trabalho ou outras atividades (p. ex.: necessitou de um esforço extra)?

\begin{tabular}{cc} 
Sim & Não \\
1 & 2 \\
1 & 2 \\
1 & 2 \\
1 & 2 \\
\hline
\end{tabular}


5. Durante as últimas 4 semanas, você teve algum dos seguintes problemas com o seu trabalho ou outra atividade regular diária, como conseqüência de algum problema emocional (como sentir-se deprimido ou ansioso?

(circule um número em cada linha)

\begin{tabular}{lcc}
\hline & Sim & Não \\
\hline Você diminuiu a quantidade de tempo que dedicava ao seu trabalho ou a outras atividades? & 1 & 2 \\
Realizou menos tarefas do que você gostaria? & 1 & 2 \\
Não trabalhou ou não fez qualquer das atividades com tanto cuidado como geralmente faz? & 1 & 2 \\
\hline
\end{tabular}

6. Durante as últimas 4 semanas, de que maneira sua saúde física ou problemas emocionais interferiram nas suas atividades sociais normais, em relação a família, vizinhos, amigos ou em grupo?

(circule um número)

\begin{tabular}{ccccc}
\hline De forma nenhuma & Ligeiramente & Moderadamente & Bastante & Extremamente \\
\hline 1 & 2 & 3 & 4 & 5 \\
\hline
\end{tabular}

7. Quanta dor no corpo você teve durante as últimas 4 semanas?

(circule um número)

\begin{tabular}{cccccc}
\hline Nenhuma & Muito leve & Leve & Moderada & Grave & Muito grave \\
\hline 1 & 2 & 3 & 4 & 5 & 6 \\
\hline
\end{tabular}

8. Durante as últimas 4 semanas, quanto a dor interferiu com o seu trabalho normal (incluindo tanto o trabalho fora de casa e dentro de casa)?

(circule um número)

\begin{tabular}{ccccc}
\hline De maneira alguma & Um pouco & Moderadamente & Bastante & Extremamente \\
\hline 1 & 2 & 3 & 4 & 5 \\
\hline
\end{tabular}

9. Estas questões são sobre como você se sente e como tudo tem acontecido com você durante as últimas 4 semanas. Para cada questão, por favor, dê uma resposta que mais se aproxime da maneira como você se sente. Em relação às últimas 4 semanas.

(circule um número para cada linha)

\begin{tabular}{lccccc}
\hline & Todo tempo & $\begin{array}{c}\text { A maior } \\
\text { parte do } \\
\text { tempo }\end{array}$ & $\begin{array}{c}\text { Uma boa } \\
\text { parte do } \\
\text { tempo }\end{array}$ & $\begin{array}{c}\text { Alguma } \\
\text { parte do } \\
\text { tempo }\end{array}$ & $\begin{array}{c}\text { Uma pequena } \\
\text { parte do } \\
\text { tempo }\end{array}$ \\
\hline $\begin{array}{l}\text { Quanto tempo você tem se sentido cheio de } \\
\text { vigor, cheio de vontade, cheio de força? }\end{array}$ & 1 & 2 & 3 & 4 & 5 \\
$\begin{array}{l}\text { Quanto tempo você tem se sentido uma } \\
\text { pessoa muito nervosa? }\end{array}$ & 1 & 2 & 3 & 4 & 5 \\
$\begin{array}{l}\text { Quanto tempo você tem se sentido tão } \\
\text { deprimido que nada pode animá-lo? }\end{array}$ & 1 & 2 & 3 & 4 & 5 \\
$\begin{array}{l}\text { Quanto tempo você tem se sentido calmo } \\
\text { ou tranqüilo? }\end{array}$ & 1 & 2 & 3 & 4 & 6 \\
\end{tabular}


Anexo 2 Continuação

\begin{tabular}{|c|c|c|c|c|c|c|}
\hline & Todo tempo & $\begin{array}{l}\text { A maior } \\
\text { parte do } \\
\text { tempo }\end{array}$ & $\begin{array}{l}\text { Uma boa } \\
\text { parte do } \\
\text { tempo }\end{array}$ & $\begin{array}{c}\text { Alguma } \\
\text { parte do } \\
\text { tempo }\end{array}$ & $\begin{array}{c}\text { Uma pequena } \\
\text { parte do } \\
\text { tempo }\end{array}$ & Nunca \\
\hline $\begin{array}{l}\text { Quanto tempo você tem se sentido com } \\
\text { muita energia? }\end{array}$ & 1 & 2 & 3 & 4 & 5 & 6 \\
\hline $\begin{array}{l}\text { Quanto tempo você tem se sentido desanimado } \\
\text { e abatido? }\end{array}$ & 1 & 2 & 3 & 4 & 5 & 6 \\
\hline Quanto tempo você tem se sentido esgotado? & 1 & 2 & 3 & 4 & 5 & 6 \\
\hline $\begin{array}{l}\text { Quanto tempo você tem se sentido uma } \\
\text { pessoa feliz? }\end{array}$ & 1 & 2 & 3 & 4 & 5 & 6 \\
\hline Quanto tempo você tem se sentido cansado? & 1 & 2 & 3 & 4 & 5 & 6 \\
\hline
\end{tabular}

10. Durante as últimas 4 semanas, quanto do seu tempo a sua saúde física ou problemas emocionais interferiram com as suas atividades sociais (como visitar amigos, parentes, etc.)?

(circule um número)

\begin{tabular}{ccccc}
\hline Todo o tempo & $\begin{array}{c}\text { A maior parte } \\
\text { do tempo }\end{array}$ & $\begin{array}{c}\text { Alguma parte } \\
\text { do tempo }\end{array}$ & $\begin{array}{c}\text { Uma pequena parte } \\
\text { do tempo }\end{array}$ & $\begin{array}{c}\text { Nenhuma parte } \\
\text { do tempo }\end{array}$ \\
\hline 1 & 2 & 3 & 4 & 5 \\
\hline
\end{tabular}

\section{O quanto verdadeiro ou falso é cada uma das afirmações para você?}

(circule um número em cada linha)

\begin{tabular}{|c|c|c|c|c|c|}
\hline & $\begin{array}{l}\text { Definitivamente } \\
\text { verdadeiro }\end{array}$ & $\begin{array}{c}\text { A maioria das vezes } \\
\text { verdadeiro }\end{array}$ & Não sei & $\begin{array}{l}\text { A maioria das } \\
\text { vezes falsa }\end{array}$ & $\begin{array}{c}\text { Definitivamente } \\
\text { falsa }\end{array}$ \\
\hline $\begin{array}{l}\text { Eu costumo adoecer um } \\
\text { pouco mais facilmente } \\
\text { que as outras pessoas }\end{array}$ & 1 & 2 & 3 & 4 & 5 \\
\hline $\begin{array}{l}\text { Eu sou tão saudável } \\
\text { quanto qualquer pessoa } \\
\text { que eu conheço }\end{array}$ & 1 & 2 & 3 & 4 & 5 \\
\hline $\begin{array}{l}\text { Eu acho que a minha } \\
\text { saúde vai piorar }\end{array}$ & 1 & 2 & 3 & 4 & 5 \\
\hline Minha saúde é excelente & 1 & 2 & 3 & 4 & 5 \\
\hline
\end{tabular}

Anexo 3 Epilepsy Surgery Inventory (ESI-55)

APPENDIX. TABLE 1. KEY TO APPENDIX

ESI-55 Scales and their item numbers

\begin{tabular}{ll}
\hline Scale Name & Item Numbers \\
\hline Health perceptions & $1,37,39,42-47$ \\
Overall quality of life & 2,48 \\
Change in health & 3 \\
Physical function & $4-13$ \\
Role limitations due to physical problems & $14-18$ \\
Role limitations due to emotional problems & $19-23$ \\
Social function & 24,34 \\
Energy/fatigue & $25,29,31,33$ \\
Emotional well-being & $26,28,30,32$ \\
Cognitive function & $35,36,38,49,50$ \\
Pain & 40,41 \\
Role limitations due to memory problems & $51-55$ \\
\hline
\end{tabular}


Anexo 3 Continuação

Appendix.

Instructions: This survey asks about your health and daily activities. Answer every question by circling the appropriate number (1, $2,3 \ldots)$. If you are unsure about how to answer a question, please give the best answer you can and write a comment or explanation in the margin. Please feel free to ask someone to assist you if you need help reading or marking the form.

1. In general, would you say your health is (circle one number):

\begin{tabular}{ll}
\hline Excellent & 1 \\
Very good & 2 \\
Good & 3 \\
Fair & 4 \\
Poor & 5 \\
\hline
\end{tabular}

2. Overall, how would you rate your own quality-of-life? Circle one number on the scale

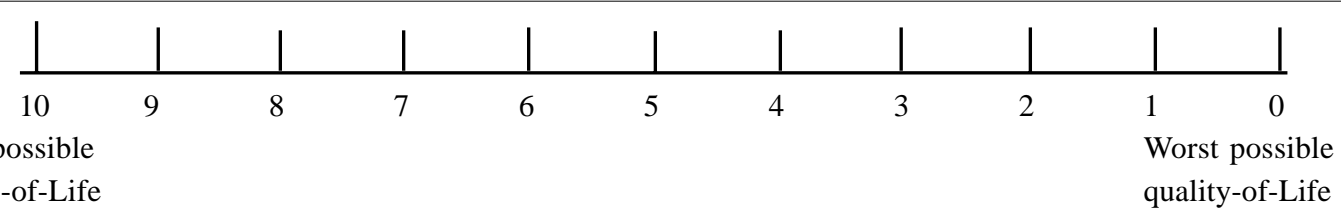

3. Compared to 1 year ago, how would you rate your health in general now? Circle one number

\begin{tabular}{ll}
\hline Much better now than 1 year ago & 1 \\
Somewhat better now than 1 year ago & 2 \\
About the same & 3 \\
Somewhat worse now than 1 year ago & 4 \\
Much worse now than 1 year ago & 5 \\
\hline
\end{tabular}

APPENDIX. TABLE 2. HEALTH AND DAILY ACTIVITIES

4-13. The following questions are about activities you might do during a typical day. Does your health limit you in theses activities? If so, how much? (Circle 1, 2, or 3 on each line)

\begin{tabular}{|c|c|c|c|}
\hline & $\begin{array}{c}\text { Yes, } \\
\text { limited } \\
\text { a lot }\end{array}$ & $\begin{array}{c}\text { Yes, } \\
\text { limited } \\
\text { a little }\end{array}$ & $\begin{array}{c}\text { No, not } \\
\text { limited } \\
\text { at all }\end{array}$ \\
\hline $\begin{array}{l}\text { 4. Vigorous activities, such as running, lifting } \\
\text { heavy objects, participating in strenuous sports }\end{array}$ & 1 & 2 & 3 \\
\hline $\begin{array}{l}\text { 5. Moderate activities, such as moving a table, } \\
\text { Pushing a vacuum cleaner, bowling, or playing Golf }\end{array}$ & 1 & 2 & 3 \\
\hline 6. Lifting or carrying groceries & 1 & 2 & 3 \\
\hline 7. Climbing several flights of stairs & 1 & 2 & 3 \\
\hline 8. Climbing one flight of stairs & 1 & 2 & 3 \\
\hline 9. Bending, kneeling, or stooping & 1 & 2 & 3 \\
\hline 10. Walking more than a mile & 1 & 2 & 3 \\
\hline 11. Walking several blocks & 1 & 2 & 3 \\
\hline 12. Walking one block & 1 & 2 & 3 \\
\hline 13. Bathing and dressing yourself & 1 & 2 & 3 \\
\hline
\end{tabular}


Anexo 3 Continuação

14-18. During the past 4 weeks, have you had any of the following problems with your regular daily activities or work as a result of any physical problems? (Please answer Yes or No for each question by circling 1 or 2 on each line)

\begin{tabular}{lcc}
\hline & Yes & No \\
\hline 14. Cut down on the amount of time you could spend on work or other activities & 1 & 2 \\
15. Accomplished less than you would like & 1 & 2 \\
16. Were limited in the kind of work or other activities & 1 & 2 \\
17. Had difficulty performing the work or other activities & 1 \\
18. Did work or other activities less carefully than usual & 2 \\
\hline
\end{tabular}

19-23. During the past 4 weeks, have you had any of the following problems with your regular daily activities or work as a result of any emotional problems (such as feeling depressed or anxious)? (Please answer Yes or No for each question by circling 1 or 2 on each line)

\begin{tabular}{lcc}
\hline & Yes & No \\
\hline 19. Cut down on the amount of time you could spend on work or other activities & 1 & 2 \\
20. Accomplished less than you would like & 1 & 2 \\
21. Were limited in the kind of work or other activities & 1 \\
22. Had difficulty performing the work or other activities & 1 \\
23. Did work or other activities less carefully than usual & 2 \\
\hline
\end{tabular}

24. During the 4 weeks, to what extent have your physical health or emotional problems interfered with your normal social activities with family, friends, neighbors, or groups? (Circle one number)

\begin{tabular}{ll}
\hline Not al all & 1 \\
Slightly & 2 \\
Moderately & 3 \\
Quite a bite & 4 \\
Extremely & 5 \\
\hline
\end{tabular}

\section{APPENDIX. TABLE 3. YOUR FEELINGS}

25-33. These questions are about how you feel and how things have you been with you during the past 4 weeks. For each question, please indicate the one answer that comes closest to the way you have been feeling. How much of the time during the past 4 weeks... (circle one number for each question)

\begin{tabular}{|c|c|c|c|c|c|c|}
\hline & $\begin{array}{c}\text { All of } \\
\text { the } \\
\text { time }\end{array}$ & $\begin{array}{l}\text { Most } \\
\text { of the } \\
\text { time }\end{array}$ & $\begin{array}{c}\text { A good } \\
\text { bit of } \\
\text { the time }\end{array}$ & $\begin{array}{c}\text { Some of } \\
\text { the } \\
\text { time }\end{array}$ & $\begin{array}{l}\text { A little } \\
\text { of the } \\
\text { time }\end{array}$ & $\begin{array}{c}\text { None } \\
\text { of the } \\
\text { time }\end{array}$ \\
\hline & 1 & 2 & 3 & 4 & 5 & 6 \\
\hline 25. Did you feel full of pep? & 1 & 2 & 3 & 4 & 5 & 6 \\
\hline 26. Have you been a very nervous person? & 1 & 2 & 3 & 4 & 5 & 6 \\
\hline 27. Have you felt so down in dumps that & 1 & 2 & 3 & 4 & 5 & 6 \\
\hline nothing could cheer you up? & 1 & 2 & 3 & 4 & 5 & 6 \\
\hline 28. Have you felt calm and peaceful? & 1 & 2 & 3 & 4 & 5 & 6 \\
\hline 29. Did you have a lot of energy? & 1 & 2 & 3 & 4 & 5 & 6 \\
\hline 30. Have you felt downhearted and blue? & 1 & 2 & 3 & 4 & 5 & 6 \\
\hline 31. Did you feel worn out? & 1 & 2 & 3 & 4 & 5 & 6 \\
\hline 32. Have you been a happy person? & 1 & 2 & 3 & 4 & 5 & 6 \\
\hline 33. Did you feel tired? & 1 & 2 & 3 & 4 & 5 & 6 \\
\hline
\end{tabular}


Anexo 3 Continuação

APPENDIX. TABLE 4.

34-39. How much of the time during the past 4 weeks...(circle one number for each question)

\begin{tabular}{|c|c|c|c|c|c|c|}
\hline & $\begin{array}{l}\text { All of } \\
\text { the } \\
\text { time }\end{array}$ & $\begin{array}{l}\text { Most } \\
\text { of the } \\
\text { time }\end{array}$ & $\begin{array}{c}\text { A good } \\
\text { bit of } \\
\text { the time }\end{array}$ & $\begin{array}{c}\text { Some of } \\
\text { the } \\
\text { time }\end{array}$ & $\begin{array}{l}\text { A little } \\
\text { of the } \\
\text { time }\end{array}$ & $\begin{array}{c}\text { None } \\
\text { of the } \\
\text { time }\end{array}$ \\
\hline 34. Has your health limited your social & 1 & 2 & 3 & 4 & 5 & 6 \\
\hline activities (like visiting with friends or close relatives? & ? 1 & 2 & 3 & 4 & 5 & 6 \\
\hline 35.Have you had difficulty concentrating or thinking? & 1 & 2 & 3 & 4 & 5 & 6 \\
\hline 36. Did you have trouble keeping your attention & 1 & 2 & 3 & 4 & 5 & 6 \\
\hline on an activity for long? & 1 & 2 & 3 & 4 & 5 & 6 \\
\hline 37. Have you worried about having another seizure? & 1 & 2 & 3 & 4 & 5 & 6 \\
\hline 38. Did you have difficulty reasoning and solving & 1 & 2 & 3 & 4 & 5 & 6 \\
\hline problems (for example making plans, making & 1 & 2 & 3 & 4 & 5 & 6 \\
\hline decisions, learning new things?) & 1 & 2 & 3 & 4 & 5 & 6 \\
\hline 39. Were you discouraged by you health problem? & 1 & 2 & 3 & 4 & 5 & 6 \\
\hline
\end{tabular}

\section{APPENDIX. TABLE 5. PAIN}

40. How much bodily pain have you had during the past 4 weeks? (Circle one number)

\begin{tabular}{ll}
\hline None & 1 \\
Very mild & 2 \\
Mild & 3 \\
Moderate & 4 \\
Severe & 5 \\
Very severe & 6 \\
\hline
\end{tabular}

41. During the past 4 weeks, how much did bodily pain interfere with your normal work (including both outside the home and housework)? (Circle one number)

\begin{tabular}{ll}
\hline Not at all & 1 \\
A little bit & 2 \\
Moderately & 3 \\
Quite a bit & 4 \\
Extremely & 5 \\
\hline
\end{tabular}

APPENDIX. TABLE 6. HEALTH IN GENERAL

42-47. Please choose the answer that best describes how true or false each of the following statements is for you. (Circle one number on each line)

42. I seem to get sick (any kind of sickness) a little easier than other people

43. I am as healthy as anybody I know

44. I expect my health to get worse

45. My health is excellent

46. When there is an illness going around, I usually catch it

47. I seem to get seizures a little easier than other people with epilepsy

\begin{tabular}{|c|c|c|c|c|}
\hline $\begin{array}{c}\text { Definitely } \\
\text { true }\end{array}$ & $\begin{array}{c}\text { Mostly } \\
\text { true }\end{array}$ & $\begin{array}{l}\text { Not } \\
\text { sure }\end{array}$ & $\begin{array}{c}\text { Mostly } \\
\text { false }\end{array}$ & $\begin{array}{l}\text { Definitely } \\
\text { false }\end{array}$ \\
\hline 1 & 2 & 3 & 4 & 5 \\
\hline 1 & 2 & 3 & 4 & 5 \\
\hline 1 & 2 & 3 & 4 & 5 \\
\hline 1 & 2 & 3 & 4 & 5 \\
\hline 1 & 2 & 3 & 4 & 5 \\
\hline 1 & 2 & 3 & 4 & 5 \\
\hline
\end{tabular}


Anexo 3 Continuação

\section{APPENDIX. TABLE 7. QUALITY OF LIFE}

48. How is the quality of your life been during the past 4 weeks, i.e., how have things been going for you? (circle one number)

\begin{tabular}{ll}
\hline Very well (could hardly be better) & 1 \\
Pretty good & 2 \\
Good and bad parts about equal & 3 \\
Pretty bad & 4 \\
Very bad (Could hardly be worse) & 5 \\
\hline
\end{tabular}

49. In the past 4 weeks, have you had any trouble with your memory? (Circle one number)

\begin{tabular}{ll}
\hline Yes, a great deal & 1 \\
Yes, somewhat & 2 \\
Yes, a little & 3 \\
No, not at all & 4 \\
\hline
\end{tabular}

50. In the past 4 weeks, have you had any trouble with your speech or language? (circle one number)

\begin{tabular}{ll}
\hline Yes, a great deal & 1 \\
Yes, somewhat & 2 \\
Yes, a little & 3 \\
No, not at all & 4 \\
\hline
\end{tabular}

51-55. During the past 4 weeks, have you had any of the following problems with your regular daily activities or work as a result of any memory, speech, or language problems? (please, answer Yes or No for each question by circling 1 or 2 on each line)

\begin{tabular}{lll}
\hline & yes & no \\
\hline 51. Cut down on the amount of time you could spend on work or other activities & 1 & 2 \\
52. Accomplished less than you would like & 1 & 2 \\
53. Were limited in the kind of work or other activities & 1 & 2 \\
54. Had difficulty performing the work or other activities & 1 & 2 \\
55. Did work or other activities less carefully than usual & 1 & 2 \\
\hline
\end{tabular}

\section{Referências}

1. Coppini RZ. Tradução, Adaptação e confiabilidade de um instrumento de avaliação de qualidade de vida para pacientes psiquiátricos graves: Lancashire Quality of Life Profile - versão brasileira. Tese apresentada à Universidade Federal de São Paulo - Escola Paulista de Medicina para obtenção do título de Mestre em Saúde Mental, São Paulo, 2001

2. Devinsky O, Penry JK. Quality of life in epilepsy: the clinician's views. Epilepsia, 34(Suppl. 4): S8-S13, 1993.

3. Santilli N, Kessler LB, Schmidt WT. Quality of life in epilepsy: persperctives of patients. In: Trimble MR, Dodson WE. (eds.). Epilepsy and quality of life. New York, Raven Press, 1994, pp. 1-17.

4. Baker GA, Jacoby A, Buck D, Stalgis C, Monnet D. Quality of life in people with epilepsy: an European study. Epilepsia, 38:353-62,1997.
5. Tureczek IE, Fandino-Franky J, Wieser HG. Comparison of the epilepsy surgery programs in Cartagena, Colombia, and Zurich, Switzerland. Epilepsia, 41(Suppl. 4):S35-S40, 2000.

6. Rao MB, Radhakrishnam K. Is epilepsy surgery possible in countries with limited resources? Epilepsia, 41(Suppl. 4):S31-S34, 2000.

7. Campos MG, Godoy J, Mesa MT, Torrealba G, Gejman $R$, Hulte I. Temporal lobe epilepsy surgery with limited resources: results and economic considerations. Epilepsia, Suppl. 4:S18-S21, 2000.

8. Wilson SJ, Bladin PF, Saling MM, Mclntosh AM, Lawrence JA. The longitudinal course of adjustment after seizure surgery. Seizure, 10(3):65-172, 2001.

9. King JT, Sperling MR, Justice AC, O'Connor MJ. A costeffectiveness analysis of anterior temporal lobectomy for intractable temporal lobe epilepsy. J Neurosurg, 87 (1):20-8, 1997. 
10. Vickrey BG. A procedure for developing a quality of life measure for epilepsy surgery patients. Epilepsia, 34 (Suppl. 4):S22-S27, 1993.

11. Vickrey BG, Hays RD, Graber J, Rausch R, Engel Jr J, Brook $\mathrm{RH}$. A health - related quality of life instrument for patients evaluated for epilepsy surgery. Medical Care, 30(4):299-319, 1992.

12. Vickrey BG, Hays RD, Rausch R, Engel Jr J, Visscher BR, Ary CM., Rogers WH, Brook R.H. Outcomes in 248 patients who had diagnostic evaluations for epilepsy surgery. Lancet, 346:1445-9, 1995.

13. Malmgren K, Sullivan M, Ekstedt G, Kullberg G, Kumlien E. Health - related quality of life after epilepsy surgery: a Swedish multicenter study. Epilepsia, 38(7):830-8, 1997.

14. Langftitt JT. Cost-effectiveness of anterotemporal lobectomy in medically intractable complex partial epilepsy. Epilepsia, 38(2):54-63, 1997.

15. Devinsky O, Cramer J. Introduction: quality of life in epilepy. Epilepsia, 34 (Suppl. 4): S1-S2, 1993.

16. Ciconelli RM. Tradução para o português e validação do questionário genérico de avaliação de qualidade de vida "Medical Outcomes Study 36 - item Short Form HealthSurvey" (SF - 36). Tese apresentada à Universidade Federal de São Paulo - Escola Paulista de Medicina para obtenção do título de Doutor em Medicina. São Paulo, 1997.

17. Fonseca PP. Um questionário para avaliação do impacto de doenças crônicas: tradução validada e estudo de confiabilidade do IEQ (Illness Effects Questionnaire). Tese apresentada à Universidade Federal de São Paulo para obtenção do título de Mestre em Saúde Mental. São Paulo 2001.

18. Carmines EG, Zeller RA. Reliability and validity assessment. Sage University Paper series on quantitative applications in social sciences. London and Beverly Hills, Sage Publications, 1979, pp.5-71.

19. Streiner DL. A checklist for evaluating the usefulness of rating scales. Can J Psychiatry, 38:140-8, 1993.

20. Cronbach LJ, MeehI PE. Construct validity in pychological testes. Psychol Bull, 52:281-302, 1955.

21. Ware JE, Gandek B. and the Iqola Project Group. The SF-36 health survey: development and use in mental health research and the Iqola Project. Int $\mathrm{J}$ Ment Health, 23:49-73, 1994

22. Hayes V, Morris J, Wolf C, Morgan M. The SF-36 health survey questionnaire: is it suitable for use with older adults? Age and Ageing, 24:120-5, 1995.

23. Wagner AK, Ehrenberg BL, Tran TA, Bungay KM, Cynn DJ, Rogers WH. Patient- based health status measurement in clinical practice: a study of its impact on epilepsy patients. Quality of Life Res 6:329-41, 1997.

24. Leidy NK, Elixhauser A, Vickrey BG, Means E, Willian MK. Seizure frequency and health-related quality of life of adults with epilepsy. Neurology, 53(1):162-6, 1999.

25. Jacoby A, Baker GA, Steen N, Buck D. The SF-36 as a health status measure for epilepsy: a psychometric assessment. Quality Life Res, 8(4):351-64, 1999.

26. Dodrill CB, Batzel LW, Queisser HR, Temkin NR. An objective method for the assessment of psychological and social problems among epileptics. Epilepsia, 21:12335,1980
27. Lorr M, Daston P, Smith IR. An analysis of mood states. Educational and psychological measurement. In: Vickrey $B G$ et al.(eds.) A health-related quality of life instrument for patients evaluated for epilepy surgery. Medical Care, 30(4):299-319, 1992.

28. Wiebe S, Rose K, Derry P. Outcome assessment in epilepsy: comparative responsiveness of quality of life and psychosocial instruments. Epilepsia, 38(4):430-8, 1997.

29. Devinsky O, Vickrey BG, Cramer JA, Perrine K, Hermann B, Meador K, Hays RD. Development of quality of life in epilepsy (QOLIE) inventory. Epilepsia, 36:1089104, 1995

30. Knut S, BjOrnaes H, Lossiun MI. Reliability and validity of a Norwegian version of the Quality of Life Epilepsy Inventory (QOLIE-89). Epilepsia, 41(1):91-7, 2000.

31. Bullinger M, Steinbüchel N. Quality of life: measurement of outcome. In: Pfäfflin M, Fraser RT, Thorbecke R, Specht V, Wolf $P$ (eds). Comprehensive Care for People with Epilepsy. London, John Libbey Ltd., 2001, pp. 27791.

32. Cramer JA, Perrine K, Devinsky, Bryant-Comstock L, Meador K, Hermann B. Development and cross-cultural translations of a 31 -item quality of life in epilepsy inventory. Epilepsia, 39(1):81-8, 1998.

33. Gillham R, Bryant-Comstock L, Kane K. Validation of the Side Effect and Life Satisfation (SEALS) Inventory. Seizure, 9:458-63, 2000.

34. Gillham R, Baker GA, Thompson P, Birbeck K, McGuire A, Tomlinson L, Eckersley L, Silveira C, Brown S. Standardisation of a self-report questionnaire for use in evaluating cognitive, affective and behavioral sideeffects of anti-epileptic drug treatments. Epilepsy Res, 24:47-55, 1996.

35. May TD, Pfäfflin M. Evaluating Comprehensive care: description of the PESOS and its psychometric properties. In: Pfäfflin $\mathrm{M}$ et al. (eds.). Comprehensive care for people with epilepsy. London, John Libbey \& Company Ltd., Eastleigh, 2001.

36. Baker GA, Jacoby A, Smith D, Dewey M. Quality of life in epilepsy: The Liverpool initiative. In: Trimble M, Dodson ED (eds.). Epilepsy and quality of life. New York, Raven Press, 1994.

37. Rapp S, Shumaker S, Gibson P, Berzon R, Hoffman R. Adaptation and evaluation of the Liverpool Seizure Severity Scale and Liverpool Quality of Life for American epilepsy patients. Qual Life Res, 7(6):467-77, 1998.

38. Baker GA, Smith DF, Dewey M, Morrow J. The development of a seizure severity scale as an outcome measure in epilepsy. Epilepsy Res, 8:245-51, 1991.

\section{Endereço para correspondência:}

Neide Barreira Alonso

Secretaria de Neurologia

Rua Pedro de Toledo, 650 - térreo, sala 1

CEP 04139-002 - São Paulo, SP

E-mail: neide.bal@uol.com.br 